\title{
Violences structurelles et violences systémiques. La violence ordinaire des rapports sociaux en Afrique
}

Jacky Bouju et Mirjam De Bruijn

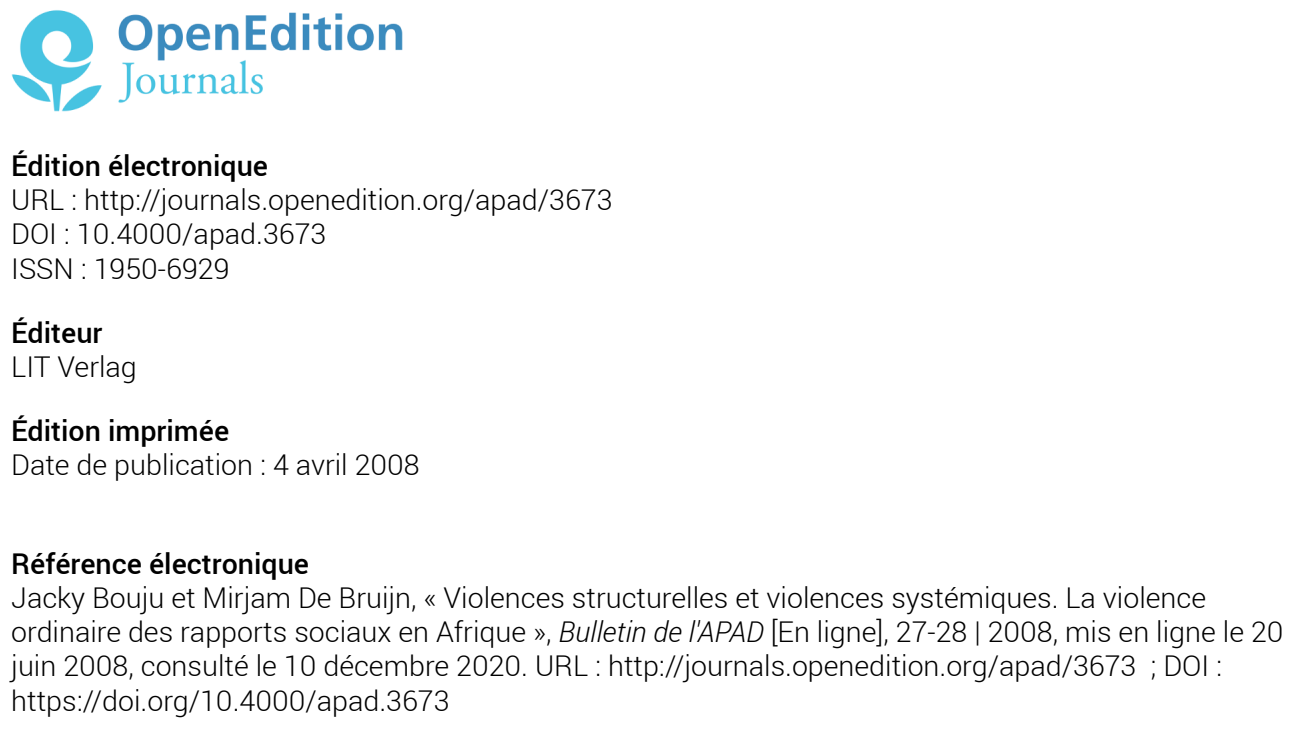

Ce document a été généré automatiquement le 10 décembre 2020.

Bulletin de l'APAD 


\title{
Violences structurelles et violences systémiques. La violence ordinaire des rapports sociaux en Afrique
}

\author{
Jacky Bouju et Mirjam De Bruijn
}

1 L'étude des violences ordinaires et quotidiennes en Afrique ne s'est développée que récemment, dans les années 1990, sous l'impulsion des ONG et des écrits militants (Bowman 2003). Cependant, les sciences sociales ne se sont guère intéressées à ce phénomène social et nous savons peu de chose de la violence ordinaire et banale qui, tout autant que la solidarité, la rivalité ou la coopération, structurent les interactions sociales quotidiennes. Les raisons du peu d'intérêt pour ce sujet semblent être de deux ordres. D'une part, le thème n'est pas facile à documenter et analyser, d'autre part les chercheurs en sciences sociales ont sans doute préféré étudier le continent africain sur des thèmes moins controversés. Par ailleurs, les études consacrées au phénomène de la violence en Afrique ont été dans l'ensemble plutôt concernées par la violence politique et, dans une moindre mesure, par la violence délinquante et l'exclusion. Ces orientations ne sont pas dues au hasard. De fait, dans le choix de ses objets, la recherche est aussi dépendante des débats qui ont cours dans les sociétés où elle s'exerce. Ainsi Sébastien Le Potvin, qui analyse la violence sociale telle qu'elle s'exprime dans la littérature malienne, montre que la violence sociale n'a jamais fait partie des problèmes à débattre dans les sphères politique et publique maliennes et la nouvelle littéraire demeure un des rares lieux d'expression collective où la violence sociale ordinaire est décrite. L'indifférence affichée de la société vis-à-vis des violences sociales est aussi soulignée par Olivier Kahola Tabu dans son étude de cas des shegue, les enfants de la rue à Lubumbashi.Pourquoi est-ce important de faire des recherches sur la violence sociale ordinaire? On peut répondre en disant que ce phénomène social ne devrait pas pas être évacué du champ de la recherche en Afrique où, comme sur les autres continents et dans différentes sociétés, la violence sociale appartient à la vie quotidienne et constitue même une des forces structurantes de la société (voir à ce sujet Abbink 2001; Abbink et al 2003). Ensuite, comme les discussions sur ce thème se sont tenues dans le cercle des ONG et des associations de militants, l'analyse du 
phénomène s'est cantonnée à un système interprétatif étroit qui a contribué à la généralisation d'une conception victimisante de l'Africain. L'intérêt des sciences sociales francophones pour la violence sociale urbaine et ordinaire en Afrique est donc fort récent. En 1998, un numéro spécial des Cahiers de l'UCAC ( $\left.\mathrm{N}^{\circ} 3\right)$ leur a été dédié et, plus récemment, en 2003, sortaient simultanément un dossier de Politique Africaine consacré à « la violence ordinaire des gens ordinaires dans des situations ordinaires » (Janin \& Marie 2003, $\mathrm{N}^{\circ}$ 91) et un Bulletin de l'APAD ( $\left.\mathrm{n}^{\circ} 25\right)$ sur « La violence endémique en Afrique" (Abéga (éd.) 2005). Ils ont permis une première description des phénomènes que nous nous proposons d'approfondir et d'analyser dans ce numéro.

Violence sociale \& contexte anomique

2 La violence sociale qui fait l'objet des articles rassemblés ici se caractérise par son caractère ordinaire, routinier et banal. Elle se distingue donc de la violence guerrière, politique ou économique qui lui est parfois liée. Elle prend place dans le quotidien de relations sociales ou d'interactions menacées par l'exploitation, l'injustice ou l'anomie de la société globale; sa compréhension confère donc un rôle important au contexte local dans lequel se déploient les rapports sociaux. Le contexte local est celui des grandes villes africaines. Celles-ci sont le produit d'une configuration historique et sociétale qui s'inscrit dans le temps long d'une histoire faite d'oppressions et de misères, marquée par les changements brutaux engendrés par la colonisation, l'instabilité politique des régimes post-coloniaux et les incertitudes économiques contemporaines. Aujourd'hui, la mondialisation de l'économie marchande accompagne la décomposition des institutions qui encadraient naguère les conduites individuelles et les processus sociaux et qui donnaient du sens aux activités humaines et à leurs conséquences. Le chômage et la misère en ne permettant plus la redistribution solidaire ont fragilisé le lien familial et augmenté la relégation. Partout, on assiste au délitement progressif du lien social et de ses solidarités alors que, simultanément, les inégalités économiques s'accroissent et que les logiques identitaires et les revendications culturelles prennent le dessus, attisant ainsi le feu des conflits urbains.

3 La généralisation de la violence sociale sous toutes ses formes a un rapport évident avec l'anomie, entendue ici au sens large comme caractéristique d'une situation où le système normatif global a perdu tout ou partie de sa légitimité, de sa rigueur et de son efficacité. L'anomie qui caractérise aujourd'hui l'ordre public régulé par le clientélisme et la corruption souligne la défaillance du pouvoir d'Etat. Les instances étatiques en pleine déliquescence ne remplissent pas leur mission de maintien de la cohésion sociale (Bouju, Kahola Tabu, Moyo). Cette situation entraîne une insoumission générale à la loi qui ne protège pas les citoyens, dont les plus pauvres n'existent guère en tant que tels mais en tant que sujets soumis à de puissants protecteurs et/ou à des puissances protectrices. Dans cette situation d'incertitude normative où les droits et les obligations cessent d'être effectivement sanctionnés, les gens ne savent pas à qui recourir pour faire valoir leurs droits quand ceux-ci sont violés, ils ne peuvent pas prévoir ce qui arrivera s'ils ne font pas ce qu'ils doivent faire et ils ne sont plus sûrs de la légitimité des obligations auxquelles ils devraient être soumis.

4 Dans ce contexte, certaines catégories sociales ne disposent pas des ressources nécessaires pour se défendre et faire face à la situation. Comme toujours, ce sont les plus pauvres et les plus faibles qui souffrent le plus. Et tout particulièrement ceux dont le statut social est dominé ou inférieur : les femmes encore et toujours (Bouju, Baker et Wallevik, Le Potvin, Ouattara) et les enfants (Kahola Tabu). Mais aussi les vieilles personnes 
(Moyo) et les hommes pauvres (Ayimpam, Chouala) dont le statut d'autorité est bafoué par la misère. Toutes ces catégories sociales quotidiennement exposées à la violence sociale risquent d'en subir des conséquences graves pour leur intégrité individuelle ${ }^{1}$.

Cependant, et ce n'est pas un des moindres paradoxes du changement social à l'œuvre, on assiste aussi à l'autonomisation des destins individuels dont l'expression plus remarquable est sans doute la possibilité d'indépendance économique des épouses, des femmes, des filles ou même des enfants que permet la ville. Ce changement de conjoncture a eu un impact profond sur les rapports de genre (Ayimpam, Chouala, Le Potvin, Moyo, Ouattara). Ainsi, la réussite économique d'épouses dont le mari est au chômage entraîne une recomposition des attentes réciproques des époux (Chouala), un réaménagement conflictuel des rôles féminins "traditionnels" (Ayimpam, Ouattara) et une "inconsistance statutaire " toujours très inconfortable à vivre pour l'homme (Moyo). Parfois, on assiste carrément à une inversion du sens habituel des échanges conjugaux violents; à tel point, qu'on a pu parler de "programmes d'ajustement domestique » dans les rapports entre conjoints (Chouala). Ainsi, selon les circonstances et les retournements de conjoncture économique, les individus se trouvent alternativement ou successivement en position dominée (victime potentielle) ou dominante (persécuteur potentiel) et inversement. De telle sorte que, selon les changements des circonstances propres à leur situation, ils exercent ou subissent tour à tour la violence sociale qu'ils contribuent ainsi à reproduire.

6 Certes, selon le contexte, certaines catégories sociales seront davantage vulnérables à la violence que d'autres. ${ }^{2}$ Certains acteurs semblent ainsi incarner la faute, l'échec ou le malheur communautaire (Baker et Wallevik, Bouju, Kahola Tabu, Ouattara). Ces catégories sociales jouent manifestement le rôle de victime émissaire en concentrant sur elles-mêmes la violence collective. Ce rapport est manifeste dans les relations entre citadins et enfants de la rue (Kahola Tabu), entre villageois et jeunes filles mères (Baker et Wallevik, Bouju), entre citadins et «bonnes à tout faire " (Bouju) mais aussi dans les excès de violence de la domination masculine ou féminine (Ayimpam, Chouala) et dans les rapports conjugaux ou parentaux (Le Potvin, Moyo, Ouattara). Le processus de victimisation ne s'épuise donc pas dans l'espace de l'événement violent et il s'avère plus dynamique, diachronique et complexe que l'acte de violence sociale ne laisse supposer. Mais en soulignant le caractère potentiellement changeant de la vulnérabilité en fonction des circonstances ${ }^{3}$ particulières dans lesquelles se trouve l'acteur, on évite l'écueil essentialiste qui consisterait à considérer la vulnérabilité à la violence comme une caractéristique stable et essentielle d'une catégorie sociale comme, par exemple, les « femmes », les « enfants » ou les « vieux ». La violence sociale apparait alors comme une dimension significative du processus de changement social qui passe, entre autres, par la recomposition négociée, imposée, ou subie des attentes réciproques constitutives de l'interaction ou du lien social.

7 Dans cet ouvrage, l'analyse du phénomène a été abordée de différents points de vue et selon différentes approches. L'approche sociologique a plutôt privilégié une analyse en termes de violence structurelle ou systémique (Chouala, Kahola Tabu, Moyo) tandis que l'approche anthropologique s'est focalisée d'une part, sur le rapport entre l'enchaînement des violences dans les trajectoires individuelles et la recomposition conflictuelles des attentes réciproques (Bouju, Le Potvin, Ouattara) et d'autre part, sur les changements de circonstances économiques dans les rapports de genre conduisant à des situations d'inconsistance statutaire génératrices de violence (Ayimpam, Moyo). 
Enfin, la violence a été abordée du point de vue de la « subjectivité » de sa constitution comme fait social à partir d'une approche herméneutique qui souligne les différences d'interprétations culturelles de ce qui fait ou non la violence d'un acte (Baker et Wallevik).

8 Mais avant tout, il convient de préciser ce qu'on désigne quand on parle de "violence sociale ». On commencera par le plus facile, à savoir l'acte violent qu'on peut définir comme tout acte intentionnel visant à assujettir autrui contre sa volonté en lui infligeant une souffrance psychique (anxiété, angoisse, désespoir, peur, c'est-à-dire tout état d'âme que l'on redoute parce qu'il a pour effet de paralyser ou de détruire tout jugement ou toute décision autonome chez l'individu) ou physique (lésions et dommages corporels). Cependant, cette définition largement acceptée de l'acte violent ne lève pas l'ambiguïté sur la nature de la violence, car, partout, on distingue entre violence et violence! La violence de l'autorité coutumière villageoise qui bannit à jamais ses jeunes filles mères est-elle de même nature que le crime de grossesse hors mariage qui les condamne? Le viol des jeunes «bonnes" par leur employeur est-il de même nature que l'infanticide pratiquée plus tard par ces mêmes jeunes filles? La violence quotidienne des enfants de la rue envers les citadins est-elle la même que celle des citadins qui ferment les yeux sur leur souffrance?

9 À l'évidence ces violences se répondent les unes les autres. Le regard du chercheur doit donc se déplacer et l'analyse changer d'objet. C'est la théorie de la violence structurelle ${ }^{4}$ qui a opéré ce déplacement du regard permettant de replacer l'acte violent dans un processus de violence dont il n'est qu'un moment remarquable. On passe ainsi de l'interaction à la structure dessinée par un cycle d'échanges violents qui a pour cadre la continuité d'un rapport social dont la violence est tout entière contenue dans l'inégalité statutaire des acteurs en présence. La théorie de la violence structurelle renvoie à la violence de la domination légale, c'est-à-dire la manière systématique dont l'autorité en place dans une structure ou une institution maintient son emprise sur une catégorie sociale (classe, groupe) en lui infligeant une souffrance psychique de sorte à entraver son émancipation. Il en va ainsi des violences domestiques ordinaires qui se développent sur le terreau fertile des représentations néotraditionnelles de l'autorité ou le châtiment corporel est érigé en technique éducative et en mode de socialisation : à la malédiction de la victime (Baker et Wallevik, Le Potvin) répond le «droit de correction du mari " (Bouju, Le Potvin) et celui de la belle-famille (Ayimpam, Chouala, Ouattara). Dans tous ces cas, la domination exercée s'appuie sur la légitimité socialement reconnue d'exercer une contrainte préventive ou punitive qui se manifeste le plus souvent par la menace de brutalité physique (coups pouvant entraîner la mort) ou de violence psychique (malédiction, répudiation, bannissement) pour rappeler les subordonnés à l'ordre social ou communautaire. La brutalité physique est la forme générale que prend la coercition en tant qu'attribut distinctif de l'autorité légitime. La brutalité engendre la peur. Souvent, la peur qui commande certains échanges familiaux est telle que l'affrontement direct est impossible, alors l'agressivité se reporte sur les plus faibles : la coépouse, l'enfant, l'aide-ménagère, le mari chômeur, la mère célibataire, la personne âgée, etc. (Ayimpam, Bouju, Le Potvin, Moyo, Ouattara).

La société globale est donc complice et elle justifie souvent les faits en invoquant le code local de l'honneur ou la coutume. L'invocation de l'honneur sert la plupart du temps à masquer la détention de pouvoirs discrétionnaires, de privilèges ou d'intérêts mercantiles dans un jeu de pouvoir local qui a pour enjeu le destin des enfants, des 
filles, des femmes et des pauvres: «Le nom, l'honneur et le sang de notre famille sont plus précieux que ta vie » (Le Potvin). La défense du point d'honneur joue aujourd'hui encore un rôle considérable dans la régulation sociale. Ce qui pose l'importante question de la légitimité du Droit dans un contexte sociétal où il n'y a guère de recours à attendre d'un Ministère de l'action sociale et de la famille dont les services sous-équipés sont absents du terrain ou dans l'incapacité réelle d'agir en dehors des priorités largement impulsées et financées par les bailleurs de fonds.

11 La dimension structurelle de la violence sociale concerne donc toutes les formes d'exploitation et d'injustice perpétrées en vue du maintien d'une domination politique, religieuse, sociale, familiale, de genre, etc. (Boute 1998 : 47). Elle est consubstantielle à la régulation de contrôle d'un ordre social et son expression soulève des problèmes de nature normative qui concernent sa légitimité et sa justification. En effet, à la coercition normative de l'ordre public (violence structurelle) ou communautaire (la violence symbolique ${ }^{5}$ ) s'opposent les violences sociales tolérées, les violences défensives (la violence systémique) et les transgressions ordinaires de l'ordre social qui en questionnent quotidiennement la légitimité. Ainsi, dans les grandes villes africaines, nombre de gens prennent leurs aises vis-à-vis des normes sociales admises ou des obligations légales. Ils violent l'ordre légal plus ou moins ouvertement en l'absence de sanction sociale ou pénale. Ce refus habituel ou occasionnel des règles qui structure le comportement collectif dans les interactions quotidiennes est aussi une forme de violence. Celle-ci a un caractère endémique, structuré par des représentations sociales et des pratiques illégales commises régulièrement par des citoyens ou des agents de l'état (en collusion) et n'impliquant pas de sanction pour les individus ou les forces de l'ordre. Ces violences créent un désordre caractéristique de la "violence systémique » (Boute 1998: 48) qui pose la question de savoir comment coopérer ensemble au maintien d'un ordre social dont la légitimité ne fait plus l'unanimité.

Un nouveau problème se pose donc à la compréhension des violences sociales ordinaires: comment faire le départ entre la violence et la coercition? Comment distinguer la mise en œuvre réglée de la contrainte (collective et légitime) chargée, entre autres, de mettre un terme à la violence en sanctionnant les violents? On pourrait répondre en considérant que dans un ordre social donné, seul le caractère légal et collectivement réglé de la coercition symbolique (sous la forme de l'impératif normatif générateur d'ordre) ou physique (sanctionnant les transgressions à cet ordre) peut faire la différence avec la violence. S'il ne fait pas de doute, en effet, que la coercition légale est le dernier rempart contre l'exploitation du faible ou le mépris de la loi commune, la confusion s'accroît encore quand on songe à la violence des exactions que peuvent commettre les forces de l'ordre «normalement " garantes de la loi. La notion de légalité est donc insuffisante et il faut faire appel à la notion de légitimité ${ }^{6}$ qui seule permet de comprendre ce qui se passe quand plusieurs ordres sociaux concurrents coexistent en parallèle dans un contexte anomique.

Violence \& discordance normative

13 Tous les cas décrits ici renvoient à des situations de discordance normative dans lesquelles un ordre peut être légal (ou coutumier) mais peu légitime ou, inversement, l'ordre social peut être légitime pour ceux qui y participent mais illégal du point de vue de la loi ou de la coutume. Sur ce point, la contribution de Jonathan Baker et Hege Wallevik et dans une moindre mesure celle de Jacky Bouju, soulignent la complexité de situations locales marquées par la discordance normative, la disjonction des légitimités 
communautaires et légales et la dysfonction des régulations de contrôle entre les différents ordres publics, communautaires villageois et sociaux urbains. Dans une telle situation, il n'est pas aisé de savoir à quoi on assiste : est-ce un acte de "violence " (forme d'interaction antisociale) ou une mesure de «coercition " (forme de contrôle social légitime).

À cet égard, Jonathan Baker et Hege Wallevik s'interrogent sur la «subjectivité » de la qualification des faits observés et de leur interprétation comme violence. En effet, du point de vue de la conception euro-occidentale des droits de l'homme qui fonde le système légal, l'acte incriminé (le bannissement des filles mères) est à l'évidence une violence perpétrée contre l'individu; mais du point de vue coutumier (Iraqw ou Bambara), le même acte est interprété comme une forme de protection d'une valeur cardinale de la communauté locale contre un acte individuel culturellement considéré comme un crime, c'est-à-dire un dommage qui affecte la société dans son ensemble. Jonathan Baker et Hege Wallevik montrent que le bannissement des jeunes femmes doit être compris comme une sanction remarquable destinée à se débarrasser de la coupable (violence structurelle) mais aussi comme un avertissement adressé à toutes les femmes et destiné à protéger la société locale contre ce qu'elle considère comme un crime. La jeune femme victime émissaire est sacrifiée pour la protection de l'intégrité de la collectivité (Ouattara). Cette problématique de Jonathan Baker et Hege Wallevik renvoie au débat sur la "subjectivité » des définitions de la violence sociale (Das \& Kleinman 1997) dans lequel on ne rentrera pas ici (car on ne sait pas ce qu'est une «subjectivité collective » en anthropologie). Mais, cela n'enlève rien au fait qu'il demeure des points de vue fondés sur des conceptions différentes de l'ordre social légitime et que le chercheur ne devrait pas esquiver la question de savoir par « qui » la violence sociale est expérimentée comme telle.

Quoi qu'il en soit, La distinction entre ces trois formes (structurelle, symbolique, systémique) de la violence sociale ordinaire permet de comprendre la dynamique par laquelle les différentes sortes de violence se transforment et se répondent les unes les autres dans le processus du changement social. Elle questionne aussi et surtout la légitimité de la coercition normative d'un ordre social très contesté et, de ce fait, largement délégitimé.

Tensions sur le lien : prises de distance \& mises à distance

Du fait même de leur caractère ordinaire, routinier ou banal, les situations de violence sociales, familiales et domestiques constituent un excellent observatoire des microchangements sociaux en train de se faire. Dans le cadre des rapports sociaux, le conflit apparaît, entre autres, comme une conséquence du travail individuel de construction identitaire des acteurs dans l'interaction. Le travail identitaire porte essentiellement sur la recomposition du cycle d'échange des attentes et des obligations réciproques. Il consiste à soutenir simultanément une tension et une cohésion entre les deux pôles contradictoires de l'engagement relationnel : le besoin de lien qui se traduit par la volonté de réduire la distance sociale (accroître l'interdépendance) et le désir d'autonomie qui se traduit par la volonté de prendre des distances (en réduisant l'interdépendance). Mais, certains acteurs sociaux n'adhèrent plus aux valeurs de l'ordre social qui les a vus naître, ils ne croient plus à la valeur de ce qu'ils sont, en termes d'identité ou de statut, ni à l'importance éminente de ce qu'on attend d'eux, en termes de rôle social. La tension initiale se manifeste alors par une disjonction ou un décalage des attentes réciproques (Bouju). Elle dégénère progressivement en conflit 
quand l'un des acteurs - le mari « fou de colère ", l'épouse « folle de rage » (Le Potvin) ou la jeune migrante urbaine (Baker et Wallevik, Bouju) - conteste la légitimité des attentes de l'autre et essaie soit de réduire la distance que l'autre veut prendre, soit de prendre une distance supplémentaire dont l'autre ne veut pas (Ayimpam, Chouala). Ces prises de distance imposées et choisies et ces mises à distances imposées ou subies signalent une difficulté quasi insurmontable à (re) négocier les termes de l'échange relationnel souvent miné par la méfiance, la suspicion, la jalousie, le ressentiment, l'envie ou la rancune qui suscitent l'indifférence à la souffrance de l'autre (Ayimpam, Kahola Tabu, Moyo, Ouattara).

Quoi qu'il en soit, le manquement systématique aux attentes de l'autre est déjà une forme de violence qui signale qu'il y a plus à gagner à sortir du cycle de l'échange social qu'à s'y maintenir. Mais en sortant du cycle de l'échange social on entre dans celui de l'échange de violence sociale. L'ensemble des textes montre qu'un enjeu majeur du conflit relationnel et de la violence sociale est soit la préservation, soit la modification du contenu effectif des obligations et des attentes de rôle qui caractérisent la situation sociale et l'identité des acteurs en interaction.

Le processus de victimisation

18 L'intérêt d'une telle approche du problème est de restituer la dynamique du cycle d'échange de la violence sociale. De ce point de vue, chaque "partenaire» peut se trouver, selon les circonstances, en position de bourreau ou de victime. Ainsi, Sylvie Ayimpam, Jonathan Baker et Hege Wallevik, Jacky Bouju, Yves A. Chouala, Fatoumata Ouattara et dans une moindre mesure Olivier Kahola Tabu ont mis l'accent sur les actes et les tactiques de résistance des acteurs en position de victime, sur les échappatoires possibles et sur leur capacité à transformer la situation dans laquelle ils se trouvent. Ces contributions montrent que la victime n'est pas sans ressource pour affronter la violence de la domination légitime même si, la plupart du temps, la résistance ne peut pas se manifester directement, ni s'exprimer de manière frontale. Elle prend alors des voies détournées: "la grève des marmites", "la grève du sexe ", "la casse domestique ", c'est-à-dire un manquement aux attentes de l'autre, une menace d'interruption de l'échange social pour tenter d'améliorer certains éléments de statut ou de rôle jugés insatisfaisants, mais sans toutefois compromettre le minimum de coopération nécessaire à la continuité de la relation sociale (Ayimpam, Chouala).

La violence ordinaire prend souvent la forme d'une victimisation multiple, d'agressions répétées et d'un harcèlement psychique continu. Elle s'inscrit dans un contexte d'insécurité sociale générale, dans des conditions spécifiques de vulnérabilité catégorielle qui transcendent les situations de violence individuelle et qui échappent à l'analyse ponctuelle (Ouattara). Mais la violence psychique est moins perceptible et plus difficile à constater que les autres formes de violence. En effet, ainsi que le souligne Yves A. Chouala, «l'intimité conjugale et l'expérience domestique en général sont le lieu de déploiement de nombreuses formes de violences silencieuses. » qui se prêtent difficilement à l'investigation. ${ }^{7}$ Ce n'est bien souvent qu'au détour de conversations, de remarques confidentielles des parents éloignés ou de commérages de voisinage qu'on en prend conscience et c'est au travers des formes visibles de l'exclusion qu'elle se manifeste : celle des "femmes maudites» (Le Potvin), ces vieilles femmes veuves chassées du village sous l'accusation de "mangeuse d'âme", ces jeunes filles mères répudiées et abandonnées à elles-mêmes (Bouju, Baker et Wallevik, Ouattara), ces jeunes paysannes vulnérables qui sont contraintes par leur famille de se louer comme 
«bonnes à tout faire» dans les grandes villes du pays afin de réunir la somme nécessaire à la constitution de leur trousseau de mariage (Bouju), ces adolescentes à peine pubères mariées contre leur gré et parfois violées par leur «fiancé ", ces femmes stériles, répudiées, ces enfants confiés aux bons soins de parents vivant en ville, dont la maltraitance quotidienne est telle qu'ils finissent par fuguer pour devenir des « enfants dans la rue » délinquants ou mendiants. L'indifférence à la souffrance de l'autre (Kahola Tabu, Ouattara) est une manifestation extrême de cette violence sociale. En laissant la parole aux "porte paroles des sans-voix » et aux "porte-voix de la parole libre » le texte de Sébastien Le Potvin peut transgresser «l'hypocrisie sociale» de la musalaka, pour atteindre «... l'impensé actuel des violences familiales » au Mali.

Et pourtant, les victimes mises à distance (bannies du village ou répudiées) arrivent à survivre à l'exclusion car elles trouvent refuge dans l'opacité sociale d'une multitude d'espaces-temps urbains de "déviance tolérée ${ }^{8}$ par la régulation de contrôle de l'ordre public. C'est dans la quotidienneté pragmatique des situations d'interaction que se construisent les écarts tolérables entre la régulation normative et la régulation effective de l'échange social. Ces écarts se traduisent par un accord partagé sur les limites des normes pragmatiques dont presque tous les acteurs sont en mesure "d'interpréter les transgressions et de savoir quand la limite des limites est dépassée " 9. À l'évidence, la formation d'espaces urbains de déviance tolérée repose sur un accord profond sur les normes effectives à ne pas transgresser mais aussi sur les transgressions acceptables par les différents ordres normatifs. Cette situation exprime sans doute une forte connivence culturelle des citadins mais celle-ci n'exclut pas pour autant l'incertitude ou la discordance normative... Et au-delà, une crise du lien social et une dégradation de la solidarité qui pose de manière aiguë la question du développement social de l'Afrique.

\section{BIBLIOGRAPHIE}

Abbink, J. \& I. van Kessel (eds), 2005, Vuanguards or Vandals, Youth, Politics and Conflict in Africa, Leiden, Brill.

Aijmer, H. \& J. Abbink (eds), 2000, Meanings of Violence, a cross cultural perspective, Oxford, NewYork, Berg.

Bowman, C.G., 2003, Theories of domestic violence in the African context, American University Journal of gender, social policy and the law, 847-863. (www.academic.udayton.edu/health/ 06world/Africa03.htm )

Boute, J., 1998, «La violence ordinaire dans les villes sub sahariennes », Cahiers de l'UCAC, $\mathrm{n}^{\circ} 3$, 39-60.

Abéga, S.C. (ed), 2003, «La violence endémique en Afrique, ", numéro special, Bulletin de l'APAD, $\mathrm{n}^{\circ} 25$.

Cahiers de L'UCAC, special issue : «Violences urbaines au sud du Sahara », Cahiers de L'UCAC, n³, 1998. 
Das, V \& A. Kleinman (eds), 2000, Violence and subjectivity, Berkeley, University of California Press.

Dubet, F., " Introduction à la problématique de la violence », Conférence Symposium de Bruxelles, source ; www.chez.com. Date de consultation 05/08/06. http ://www.chez.com/b105/ lectures/dubet/violence.htm

Janin P.\& A. Marie, (eds), 2003, « Violences ordinaires », numéro special, Politique africaine, $\mathrm{n}^{\circ} 91$.

\section{NOTES}

1. Les formes d'adaptation à la souffrance et d'intériorisation de la vulnérabilité sont mal connues. La schizophrénie en est une qui, du point de vue phénoménologique, peut être envisagée « comme un mode particulier d'adaptation à la fois psychique et social mobilisé face à un environnement ressenti comme profondément hostile. » Elle représente une forme de déplacement ou de rupture du point d'équilibre du processus continu de construction et de maintien de la « carte interne susceptible de nous informer au sujet de qui nous sommes, de comment marche le monde et de comment nous entrons en rapport avec ce monde pour gérer la complexité de notre existence et de notre croissance » (Strauss, J.S. 1997, «La nature de la schizophrénie : vulnérabilité et destin ", L'évolution psychiatrique, 62, $2: 245-262): 260$ ).

2. Chaque registre d'inégalité sociale fondé sur la richese, le genre, l'aînesse, la classe sociale, l'honneur, etc., constitue un espace de vulnérabilité spécifique. Les jeunes migrantes paysannes cumulent les risques de violence car elles se trouvent à l'intersection de ces différents espaces vulnérabilité.

3. Une situation est faite de l'ensemble des relations concrètes qui, à un moment donné, unissent un individu ou un groupe aux circonstances dans lesquelles il doit vivre ou agir.

4. Concept apparu dans les années soixante-dix et communément attribué au sociologue pacifiste suédois Johan Galtung.

5. La violence symbolique est la dimension culturelle ou communautaire de la violence structurelle, elle écarte toute solution alternative pour ne garder que la possibilité proposée par l'ordre social local comme allant de soi et seule valable.

6. Définie ici comme la croyance collective à l'excellence des valeurs, des normes et des règles d'un ordre social partagé.

7. Alors que les études de cas qui constitueront ce numéro s'appuient sur des enquêtes de terrain, le texte de Sébastien Le Potvin qui passe en revue les représentations de la violence sociale dans la littérature malienne fait exception. Son intérêt, majeur, était de permettre un dépassement du silence que les règles de bienséance et de pudeur imposent à l'entretien.

8. Selon George Dubet, la déviance tolérée repose sur une injonction elle-même paradoxale qui consiste à affirmer nettement les interdits, tout en concédant des moments, des lieux et des formes dans lesquels ces interdits peuvent être transgressés ; plus encore, dans lesquels il est implicitement souhaitable que ces interdits soient transgressés (Dubet, Introduction...).

9. Dubet, «Ibid. ». 


\section{RÉSUMÉS}

L'étude de la violence ordinaire s'est développée dans les années 90, principalement dans les cercles d'ONGs et de mouvements militants. Pour les sciences sociales, c'est un nouveau terrain de recherche qui a besoin d'investigations approfondies. Dans cette introduction du bulletin de l'APAD sur les "violences ordinaires", nous explorons différentes réflexions théoriques sur l'étude de la violence au quotidien. Un des principaux arguments est que la violence actuelle, telle que décrite dans les différentes études de cas, peut être liée aux changements qui affectent les rapports sociaux autant que les normes et valeurs dans des sociétés africaines en mutation accélérée. Cependant, en l'état actuel de nos connaissances, la prudence s'impose et nous incite à éviter toute perspective victimisante dans l'analyse de la violence du changement social en Afrique.

Title : Structural Violences \& Systemic Violences. Daily Violence in African Social Links The study of ordinary violence only developed in the 1990ies, mainly in circles of NGO and militant writing. For the social sciences it is a new field of study that still needs to be explored. In this introduction of the special issue of the APAD bulletin on 'violences ordinaires' we explore different theoretical reflections on the study of ordinary violence. One of the principal arguments is that the present day violence as described in the different case studies can be linked to changes in social relations and norms and values surrounding these in African societies who are rapidly transforming. However we should be cautious in our presentations and avoid victimisation perspectives in the analysis of social change in Africa in terms of violence. 